\title{
Edge-based formulation of elastic network models
}

\author{
Maxwell Hodges $\odot,{ }^{1,{ }^{*}}$ Sophia N. Yaliraki, ${ }^{1}$ and Mauricio Barahona ${ }^{2, \dagger}$ \\ ${ }^{1}$ Department of Chemistry, Imperial College London, London SW7 2AZ, United Kingdom \\ ${ }^{2}$ Department of Mathematics, Imperial College London, London SW7 2AZ, United Kingdom
}

(Received 2 July 2019; published 31 December 2019)

\begin{abstract}
We present an edge-based framework for the study of geometric elastic network models to model mechanical interactions in physical systems. We use a formulation in the edge space, instead of the usual node-centric approach, to characterize edge fluctuations of geometric networks defined in $d$-dimensional space and define the edge mechanical embeddedness, an edge mechanical susceptibility measuring the force felt on each edge given a force applied on the whole system. We further show that this formulation can be directly related to the infinitesimal rigidity of the network, which additionally permits three- and four-center forces to be included in the network description. We exemplify the approach in protein systems, at both the residue and atomistic levels of description.
\end{abstract}

DOI: 10.1103/PhysRevResearch.1.033211

\section{INTRODUCTION}

Elastic network models (ENMs) are ubiquitous in physics and have been applied to describe properties of a wide variety of structures including glasses [1,2], biological tissue [3], supercooled liquids [4], and, recently, the design of allosteric materials [5]. A particularly useful application of ENMs, sparked by the seminal work of Tirion [6], has been in the study of protein structures, as the use of molecular dynamics (MD) simulations on biologically relevant timescales remains challenging. The principal assumption of ENMs is that we may approximate the bottom of the potential energy well of a structure by a quadratic function, by taking the Taylor series of the potential energy with respect to node displacements about the minimum $\mathbf{r}_{0}$. In elastic models, the forces $\mathbf{f}$ are thus linear in the displacements $\mathbf{r}$, i.e., $\mathbf{f}=\mathbf{H}\left(\mathbf{r}_{\mathbf{0}}\right)\left(\mathbf{r}-\mathbf{r}_{0}\right)$, where $\mathbf{H}\left(\mathbf{r}_{\mathbf{0}}\right)$ is the Hessian matrix obtained by differentiating twice the potential function. Typically, the analysis of (infinitesimal) motions involves diagonalization of $\mathbf{H}$ to determine the normal modes of the protein. While real potential energy surfaces of proteins are complex, highly nonlinear, and containing many minima [7], elastic models have been surprisingly effective for the analysis of slow equilibrium motions of proteins $[8,9]$. Another common use of ENMs is for the calculation of node fluctuations, which have shown good agreement with crystallographic B factors $[10,11]$.

The focus of ENMs has thus typically been on the node variables. Here, we present an edge-based formulation of ENMs, which instead puts the emphasis on the interactions

\footnotetext{
*m.hodges14@imperial.ac.uk

†m.barahona@imperial.ac.uk
}

Published by the American Physical Society under the terms of the Creative Commons Attribution 4.0 International license. Further distribution of this work must maintain attribution to the author(s) and the published article's title, journal citation, and DOI. between the nodes, which in a mechanical framework corresponds to extensions (or compressions) of the "springs" associated with the edges. More formally, the edge changes are the dual of the node motions [12]. An edge-centric approach has proved highly effective in previous studies of different networks [13-15], and indeed the formulation presented need not be restricted to proteins and is general for networks embedded in $d$-dimensional space. There has been extended discussion in the literature over the use of networks with scalar node variables to model two- and three-dimensional mechanical structures [16]. By instead working in edge space, we avoid this issue altogether since the scalar edge variables, which represent changes in edge length, appear naturally in the theory regardless of the dimensionality of the geometric structure. Historically, the Born-Huang model [17] has often formed the basis for the study of lattice structures but its weakness in handling disordered materials like glasses has been highlighted in the context of rigidity percolation [18] and more recently by Zaccone and Scossa-Romano [19], who extended the Born model to include nonaffine responses to external stresses. In many systems such as proteins, function is often driven by changes in structure, but crucially it is the relative change in node positions that is of interest. We thus show how to obtain edge fluctuations in elastic network models and compute the edge mechanical embeddedness as a useful property of the system. Finally, we show how this formulation naturally connects with the rigidity properties of the network, viewed as a set of edge constraints. We showcase the approach with specific protein examples.

\section{THEORY}

\section{A. Edge-based formulation of geometric elastic network models}

Consider a network of $N$ nodes, associated with points in $d$-dimensional space $\mathbf{r}_{i, 0} \in \mathbb{R}^{d}$, and with $E$ interactions between nodes (due to, e.g., physicochemical potentials). Let us denote the (small) node displacements as the $d$-dimensional 


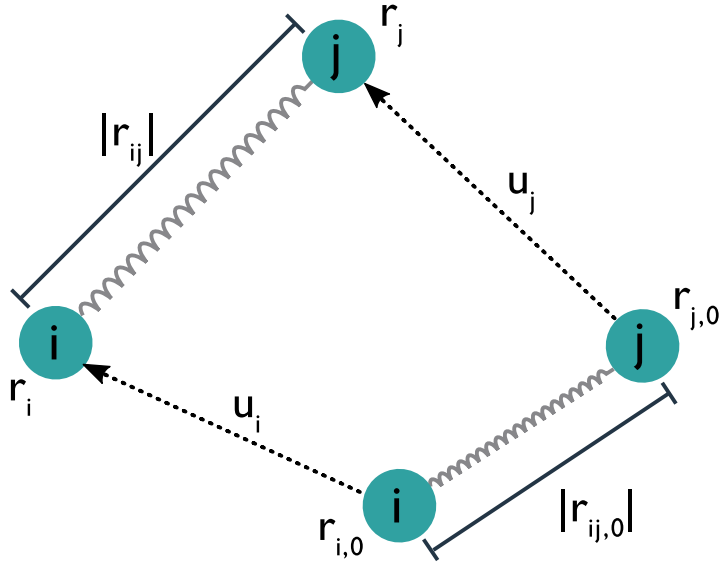

FIG. 1. The extension of the spring can be written in terms of the displacements of the nodes [12].

vectors

$$
\mathbf{u}_{i}=\mathbf{r}_{i}-\mathbf{r}_{i, 0}, \quad i=1, \ldots, N .
$$

Each edge has an associated scalar variable $e_{\alpha} \in \mathbb{R}, \alpha=$ $1, \ldots, E$, which measures its extension, i.e., its change in length. The node and edge variables are related directly through the $N d \times E$ geometric incidence matrix $\mathcal{B}$ :

$$
\mathbf{e}=\mathcal{B}^{T} \mathbf{u}
$$

where $\mathbf{u}^{T}=\left(\mathbf{u}_{1}^{T}, \ldots, \mathbf{u}_{N}^{T}\right)$ is the $N d$-dimensional vector compiling the node displacements, and $\mathbf{e}$ is the $E$-dimensional vector of edge extensions.

To obtain the form of the geometric incidence matrix, note that each column of $\mathcal{B}$ is associated with an edge. Assuming small node displacements, it can be easily shown (Fig. 1 and Appendix A) that the extension of the edge $\alpha=(i j)$ between nodes $i$ and $j$ induced by the node displacements $\mathbf{u}$ [to order $O\left(|\mathbf{u}|^{2}\right)$ ] is given by

$$
e_{\alpha}=\left|\mathbf{r}_{i j}\right|-\left|\mathbf{r}_{i j, 0}\right| \simeq \widehat{\mathbf{r}}_{i j}^{T}\left(\mathbf{u}_{j}-\mathbf{u}_{i}\right),
$$

where $\widehat{\mathbf{r}}_{i j}=\mathbf{r}_{i j, 0} /\left|\mathbf{r}_{i j, 0}\right|=: \widehat{\mathbf{r}}_{\alpha}$ is the $d$-dimensional unit vector along the direction of edge $\alpha=(i j)$. Each row of $\mathcal{B}^{T}$ is a vector $\mathcal{B}_{\alpha}^{T}$ that follows from Eqs. (2) and (3):

$$
\begin{aligned}
\mathcal{B}_{\alpha}^{T} & =\left(0, \ldots, 0,-\widehat{\mathbf{r}}_{\alpha}^{T}, 0, \ldots, 0, \widehat{\mathbf{r}}_{\alpha}^{T}, 0, \ldots, 0\right), \\
\alpha & =1, \ldots E
\end{aligned}
$$

to form the geometric incidence matrix: $\mathcal{B}=\left(\mathcal{B}_{1} \ldots \mathcal{B}_{E}\right)$. Note that the matrix $\mathcal{B}$ is akin to the standard $N \times E$ incidence matrix $B$ in graph theory [14] but it includes full directional information through the $d$-dimensional edge unit vectors.

Invoking a mechanical description, we can use Hooke's law and Newton's third law to obtain the usual linear relationship between input forces on the nodes $\mathbf{f}_{\text {nodes }}$ and the induced node displacements $\mathbf{u}$ :

$$
\mathbf{K u}=\mathbf{f}_{\text {nodes }},
$$

where $\mathbf{f}_{\text {nodes }}$ is the $N d \times 1$ vector compiling the external forces on the nodes and $\mathbf{K}$ is the $N d \times N d$ stiffness matrix

$$
\mathbf{K}=\mathcal{B} \mathbf{G} \mathcal{B}^{T},
$$

with $\mathbf{G}=\operatorname{diag}\left(g_{\alpha}\right)$ denoting the $E \times E$ diagonal matrix of spring constants. The stiffness matrix is thus the Hessian of the system, indeed this is the only form the Hessian can take [16].

Using our formulation, we can study the input-output properties of the system in terms of edge variables, i.e., the edge extensions $\mathbf{e}_{\text {out }}$ induced by external forces $\boldsymbol{f}_{\text {in }}$ applied to the edges. Let us consider external forces applied along the edges, which we compile in an $E \times 1$ vector $f_{\text {in }}$. These edge forces result in edge compressions and stretches that induce forces on the nodes given by

$$
\mathbf{f}_{\text {nodes }}=\mathcal{B} \boldsymbol{f}_{\text {in }} .
$$

We wish to disregard any components of the induced forces linked to rigid motions of the elastic network since such motions do not produce edge extensions $\left(\mathbf{e}_{\text {out }}=0\right)$. This can be achieved naturally by considering the pseudoinverse of the stiffness matrix. The induced nonrigid displacements are given by

$$
\mathbf{u}^{+}=\mathbf{K}^{+} \mathcal{B} \boldsymbol{f}_{\text {in }},
$$

where $\mathbf{K}^{+}$is the Moore-Penrose pseudoinverse of $\mathbf{K}$, and the edge extensions induced by the applied edge forces are given by

$$
\mathbf{e}_{\text {out }}=\mathcal{B}^{T} \mathbf{K}^{+} \mathcal{B} \boldsymbol{f}_{\text {in }}=: \mathbf{T} \boldsymbol{f}_{\text {in }} .
$$

For the input force $\boldsymbol{f}_{\text {in }}$, the output vector $\mathbf{e}_{\text {out }}$ records the induced change in length of all the edges in the network. The meaning of the $E \times E$ matrix $\mathbf{T}$ is clear: given a unit force (input) applied along edge $\alpha$, the induced (output) extension at edge $\beta$ is the corresponding entry of $\mathbf{T}$ :

$$
e_{\beta}=\left(\mathcal{B}_{\beta}\right)^{T} \mathbf{K}^{+} \mathcal{B}_{\alpha}=T_{\beta \alpha} .
$$

As a consequence, the induced extension at the input edge $i$ is given by the diagonal element $T_{\alpha \alpha}$, which, depending on the location of the spring within the network, might not necessarily be the same as if the spring was isolated. This is the mechanical analog of the effective resistance in electrical networks [14,20], also known as the resistance distance [21], yet, in our case, it is both the connectivity and the geometry of the network in $d$-dimensional space that determines edge responses. We exploit this concept in the following section through the definition of the edge mechanical embeddedness.

\section{B. Edge fluctuations and mechanical embeddedness}

One application of the model is to identify residue-residue interactions within a protein that exhibit the highest edge fluctuations. To see this, consider the Langevin equation of a three-dimensional elastic network $(d=3)$ representing protein residues undergoing dynamical motion in a heat bath modeling the aqueous environment:

$$
\mathbf{M} \frac{d^{2} \mathbf{r}}{d t^{2}}+\Gamma \frac{d \mathbf{r}}{d t}+\mathbf{K}\left(\mathbf{r}-\mathbf{r}_{0}\right)=\eta(t),
$$

where $\mathbf{M}$ is a diagonal mass matrix, $\boldsymbol{\Gamma}$ is the diagonal damping matrix, and $\eta(t)$ is a vector of independent and identically distributed (i.i.d.) Gaussian noises. The damping terms arise from interactions of the protein with water and itself, and are typically large. Hence, we consider the overdamped limit, where we may neglect inertial terms. Although larger damping is sometimes set for residues located deeper inside the 
structure [22], for simplicity we set all damping values to be equal and we renormalize time to obtain

$$
\frac{d \mathbf{r}}{d t}=-\mathbf{K}\left(\mathbf{r}-\mathbf{r}_{0}\right)+\boldsymbol{\eta}(t),
$$

which has the general solution

$$
\mathbf{u}(t)=\mathbf{r}(t)-\mathbf{r}_{0}=\int_{-\infty}^{t} \exp [\mathbf{K}(t-s)] \boldsymbol{\eta}(s) d s,
$$

where the residue position $\mathbf{r}(t)$ is now a random variable. We are again interested in the random fluctuations of the edge extensions (2). Utilizing our geometric incidence matrix, one can show that the covariance matrix of the edge fluctuations is given by

$$
\mathbb{E}\left[\mathbf{e}(t) \mathbf{e}(t)^{T}\right]=\frac{1}{2} \mathcal{B}^{T} \mathbf{K}^{+} \mathcal{B}=\frac{1}{2} \mathbf{T} .
$$

In a number of papers, authors construct networks from residue-residue interactions and identify significant residues using measures of centrality, such as edge or node betweenness [23-25]. However, it is not clear what the physical significance of such measures is. In contrast, the mean edge fluctuations are related to a graph-theoretical measure called edge embeddedness, first introduced in Ref. [14] in the context of random walks on networks and resistor networks. We may then define the equivalent mechanical embeddedness for edge $\alpha$ in a geometric elastic network in $d$ dimensions as

$$
\varepsilon_{\alpha}=1-\left(\mathbf{G} \mathcal{B}^{T} \mathbf{K}^{+} \mathcal{B}\right)_{\alpha \alpha}=1-g_{\alpha} T_{\alpha \alpha} .
$$

The mechanical embeddedness has a clearer physical meaning: the second term is the fraction of the input force applied to edge $\alpha$ that edge $\alpha$ actually feels. If an edge feels all the force applied to it, it is not well "embedded" within the network and has a low value of $\varepsilon$ (i.e., it is not strongly coupled to the rest of the network and does not dissipate its fluctuations into the network). Conversely, edges that are more "embedded" within the network structure feel a lower force, dissipate fluctuations into the rest of the network, and have a larger $\varepsilon$ score nearer to 1 .

\section{Connection to infinitesimal rigidity}

There is also a straightforward relationship between the geometric incidence matrix $\mathcal{B}$ and the classic rigidity matrix $\mathbf{R}$ of the structure, given in Eq. (D5) [26]. In Appendix D, we show that

$$
\mathcal{B}^{T}=\mathbf{D}^{-1} \mathbf{R},
$$

where $\mathbf{D}$ is the $E \times E$ diagonal matrix containing the interaction distances. The rigidity matrix can be used to determine the rigid parts of the elastic network structure (i.e., those that allow no internal motion) and the flexible parts via the concept of infinitesimal rigidity. (The distinction between rigidity and infinitesimal rigidity is discussed in depth in Ref. [26], but here we consider only generic structures and so the two terms are equivalent.) The rigidity matrix of a three-dimensional structure possesses six zero eigenvalues corresponding to three translations and three rotations, but may have additional zero eigenvalues associated with motions of the structure that lead to no change in the potential energy of springs in the network. In Appendix E, we summarize an infinitesimal rigidity algorithm developed in Ref. [27] that uses the set of eigenvectors associated with such additional zero eigenvalues (if they exist) to cluster the structure into rigid clusters. At the cost of longer running times, this infinitesimal rigidity algorithm allows greater flexibility in the choice of constraints than the popular rigid cluster decomposition based on FIRST $[28,29]$, which is computationally efficient, yet it imposes the presence of angle constraints in the network structure.

In some systems, such as chemical bonds within molecules, we do in fact have additional constraints on the angles between edges. Indeed, inclusion of three-center interactions in the simulation of polymer glasses has been shown to be important for the interpretation of Raman scattering spectra [30]. We therefore consider three-center interactions (and indeed four-center interactions, corresponding to dihedral angles). Given three nodes $i, j, k$ with edges $(i j)$ and $(j k)$, we compute the change in length of edge $(i k)$ with the constraints that the other two edges are held constant: $\left|\mathbf{r}_{i j}\right|^{2}=\left|\mathbf{r}_{i j, 0}\right|^{2}$ and $\left|\mathbf{r}_{j k}\right|^{2}=\left|\mathbf{r}_{j k, 0}\right|^{2}$. Expanding these equations and substituting into the expression for the extension of edge $(i k)$, which is opposite to node $j$, we obtain (see Appendix B)

$$
e_{i k}=\frac{1}{\left|\mathbf{r}_{i k, 0}\right|}\left(\mathbf{r}_{j k, 0}^{T}, \quad\left(\mathbf{r}_{i j, 0}^{T}-\mathbf{r}_{j k, 0}^{T}\right), \quad-\mathbf{r}_{i j, 0}^{T}\right)\left(\begin{array}{c}
\mathbf{u}_{i} \\
\mathbf{u}_{j} \\
\mathbf{u}_{k}
\end{array}\right) .
$$

Note that the three-center extension (15) relative to the "angle" at node $j$ is not the same as if a two-center Hooke spring was placed between nodes $i$ and $k$. From expressions of the form (15), we can construct the three-center stiffness matrix $\mathbf{K}_{\text {angle }}$.

Using a similar procedure, we also find the expression for the linear changes of a four-center interaction, by keeping the three two-center and two three-center interactions constant. Such changes lead to the four-center stiffness matrix $\mathbf{K}_{\text {dihedral }}$. See Appendix C.

The total stiffness matrix is then the sum of the stiffness matrices: $\mathbf{K}_{\text {total }}=\mathbf{K}_{\text {bond }}+\mathbf{K}_{\text {angle }}+\mathbf{K}_{\text {dihedral }}$, where $\mathbf{K}_{\text {bond }}$ is the two-center matrix given in Eq. (6). The extensions $\mathbf{e}_{\text {out }}$ induced by input forces $\boldsymbol{f}_{\text {in }}$ follow the same form as in Eq. (7):

$$
\mathbf{e}_{\text {out }}=\mathcal{B}^{T} \mathbf{K}_{\text {total }}^{+} \mathcal{B} f_{\text {in }}=: \mathbf{T}_{\text {total }} f_{\text {in }} .
$$

Below, we study the effect of the different components of the stiffness matrix in the input-output properties of the system.

\section{APPLICATIONS}

\section{A. A mechanical model of protein-ligand binding at the atomistic level}

Allostery is a biological process whereby the binding of a ligand to a protein leads to a functional change at a distant site (often the active site) of the protein [31,32]. A common explanation for allostery is that ligand binding leads to a propagation of strain across the protein structure, potentially along specific residue pathways, causing a structural change at the active site.

Here, we study this process using an atomistic elastic network model of a protein bound to an allosteric ligand. First, we measure the elastic response elicited across the protein by the application of unit forces to all weak interactions between the ligand and the protein allosteric site with 
TABLE I. Spring constants for each of the elastic network interactions.

\begin{tabular}{lc}
\hline \hline Interaction & Spring constant \\
\hline Covalent & 100 \\
Hydrogen & 10 \\
Hydrophobic & 1 \\
Angle & 1 \\
Dihedral & 0.1 \\
\hline \hline
\end{tabular}

negative forces corresponding to compressions of the source interactions and positive forces corresponding to extensions (although the overall sign is arbitrary). Furthermore, we apply infinitesimal rigidity analysis (Appendix E) to obtain the rigid clusters within the protein to elucidate the propagation of the strain. Since strain cannot propagate through floppy regions, we expect both the allosteric site and active sites to be within the same rigid cluster if strain is to pass from one site to the other efficiently.

Atomistic graphs are constructed from PDB files containing full three-dimensional (3D) atomic data of protein structures, and the software FIRST [29] to determine the presence of the various bond types (covalent, hydrogen, and hydrophobic interactions). We assign values to the spring constants of the edges with the correct order of magnitude, as per the Amber15fb force field [33] (Table I). We do not use exact values for each interaction since it is difficult to assign spring constants to hydrogen bonds and hydrophobic interactions from force fields used in molecular dynamics. In such fields, hydrophobic interactions emerge from the presence of implicit or explicit water that favors interactions to polar regions of the protein, whereas hydrogen bonds are derived from electrostatic contributions.

We obtain the output extension for all edges in the protein in response to inputs at source edges given by the interactions with the ligand, and exemplify the results through the allosteric protein PDK1 (PDB code: 3ORZ). In Fig. 2 we show the top $2 \%$ of bonds by absolute length change (i.e., we do not
Bond results with bonds only

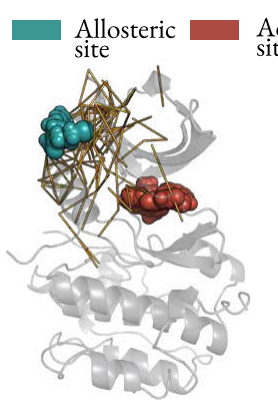

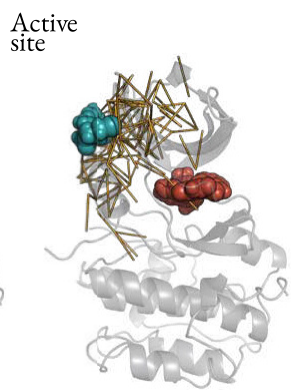

Bond results with bonds and angles

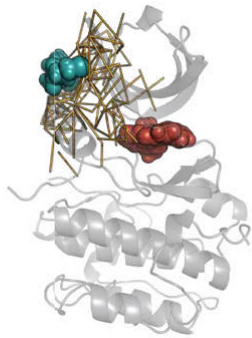

Bond results with bonds, angles and dihedrals
FIG. 2. Elastic response of PDK1 (PDB code: 3ORZ [34]). The top $2 \%$ of bonds by absolute extension are shown for three cases: (left) only the two-center interactions (bonds) are included in the network; (center) three-center (angle) interactions are added to the network and the top output extensions of bonds are shown; (right) dihedral angles are added and again only top output bond displacements are shown.

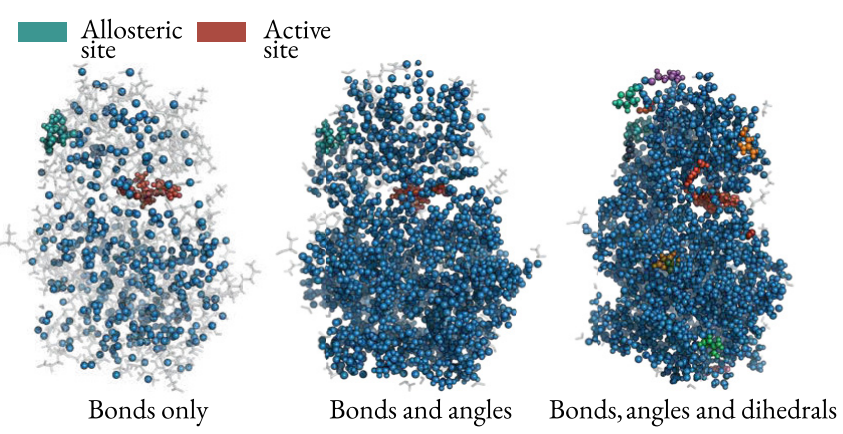

FIG. 3. Infinitesimal rigidity of PDK1. Each cluster has a different color and "floppy" (nonrigid) atoms are shown in transparent gray: (left) only bonds included as constraints, leading to a single large cluster in blue with all other atoms floppy; (center) angle constraints added; (right) dihedral constraints also added leading to a large rigid cluster extending over the whole protein. The rigid clusters and floppy atoms are computed using the algorithm in Appendix E, which was introduced in Ref. [27].

discriminate between bond stretching or compression) in three scenarios: (i) where only the two-center bond interactions are used to construct the elastic network, as is traditionally the case with elastic networks of proteins; (ii) where angle constraints between pairs of covalent bonds are included; and (ii) where dihedral angle constraints from double bonds are also modeled. Given that the highest scoring interaction in the bonds-only network (i) (the hydrophobic interaction between Lys120 and Asn122) exhibits an extension of 0.766, we choose to represent the top $2 \%$, which exhibit changes above 0.01 , as a reasonable cutoff. The most stretched edges are all located in the area connecting the allosteric and active sites. Furthermore, the infinitesimal rigidity analysis (Fig. 3) shows that, even for the two-center stiffness matrix, the allosteric site and the region around the active site (Va196, Lys111, Tyr161, Ala162, Thr222, Asp223) all appear in a rigid cluster, with Leu88 the only active site residue that has no atoms within the rigid cluster. When three- and four-center constraints are included, the protein becomes strong due to the qualitative nature of the infinitesimal rigidity condition. It appears then plausible that propagation of strain may be emitted from binding at the allosteric site toward the active site, particularly through the rigid cluster formed by the two-center interactions that contains a smaller subset of the atoms in the protein.

The results of the elastic response show that the $\mathbf{e}_{\text {out }}$ decrease exponentially with distance (correlation coefficient $=-0.603$ ), even when angles and dihedrals are included (see Fig. 8). Such a response is similar to random networks [5] and is not suggestive of a structure exclusively optimized for directed perturbations. Indeed, the two largest extensions are found in the Lys120-Asn122 (0.766) and Val124-Pro125 (0.437) hydrophobic interactions, which are within $5 \AA$ of the allosteric source site, whereas the active site is around $17 \AA$ away. The highest scoring interactions involving active site residues are two Lys111-Phe157 hydrophobic interactions, which have extensions of 0.0122 , and rank 130th and 131st. Of the top 2\% interactions (149 out of a total of 7391 edges) by output extensions, all but 4 are hydrophobic interactions. This is unsurprising given they have the weakest spring 
constants, but appears to lead to those weak interactions near the allosteric site effectively acting like a sponge, absorbing the shock of input forces and preventing long-range transfer of displacement. If we change the force constant of the hydrophobic interactions to 10 (the same as the hydrogen bonds), the range of the propagation increases. However, it is difficult to rationally assign such spring constant values to the hydrophobic interactions, and there does not appear to be strong evidence that the allosteric effect exhibited by PDK1 is mediated by traversal of strain energy. We have performed the elastic response analysis on a further two proteins (h-Ras, ATCase) with similar results. Hence, our examples indicate that topological notions alone (such as rigidity) do not fully determine if a mechanical explanation for allostery is plausible, as the particular values of the edge spring constants are also crucial.

\section{B. Fluctuations of residue-residue interactions}

We applied our edge-based geometric formulation to a residue-residue interaction network (RRIN), i.e., an elastic network model of a protein at the residue level. We constructed several RRINs for ADK (4AKE [35]) using different distance cutoffs $(7,10,12$, and $15 \AA)$ and obtained the average displacement for each of the edge interactions (12). To decide on the appropriate cutoff, we computed Spearman's correlation coefficient $(\rho)$ of the resulting extensions across the RRINs created with different cutoffs and found greater robustness for larger cutoff values: $\rho=0.216$ between the 7 and $10 \AA$ RRINs; increasing to $\rho=0.679$, between the 10 and $12 \AA$ RRINs; and increasing further to $\rho=0.801$ between the RRINs created with 12 and $15 \AA$ cutoffs. (Below $7 \AA$, zeroenergy modes appear in the network as revealed by singular value decomposition of the rigidity matrix $R$.) We thus use a cutoff of $12 \AA$ here and a single force constant (arbitrarily set to 1 ), in line with other reports in the literature [36].

Figure 4 shows the results of our analysis of the this RRIN of open $\mathrm{ADK}$ (4AKE). A relatively right-skewed distribution of edge displacement values is observed (Pearson median skewness $=0.580$ ), with average value of 0.236 and a number of interactions scoring significantly highly. The top $2 \%$ of interactions by rank are those scoring above 0.409 and the top $1 \%$ score above 0.452 . The most highly scoring interactions are clustered primarily in the lid and $\mathrm{AMP}_{\text {bind }}$ domains, corresponding closely to those regions of the protein that are structurally altered during the open-to-closed transition. Qualitatively similar observations were obtained by Mitchell et al. [37] from dynamic data, i.e., by comparing residue displacements across an NMR ensemble of structures to calculate local strain. Note that here, however, just a single structure is used and strain is predicted a priori, emphasizing the fact that the intrinsic topology of the protein determines where strain is distributed to assist function. The highest scoring interaction with 0.701 is Gly56-Lys57; Lys57 is one of the residues that shifts more than $10 \AA$ during the open-to-closed transition [38] while Gly56 has been shown to display particularly high fluctuations in coarse-grained MD simulations at the residue level [39]. Since we follow standard convention and use the same force constant for all residue-residue interactions (arbitrarily set to 1), by Eq. (13) (with $G$ equal to the identity

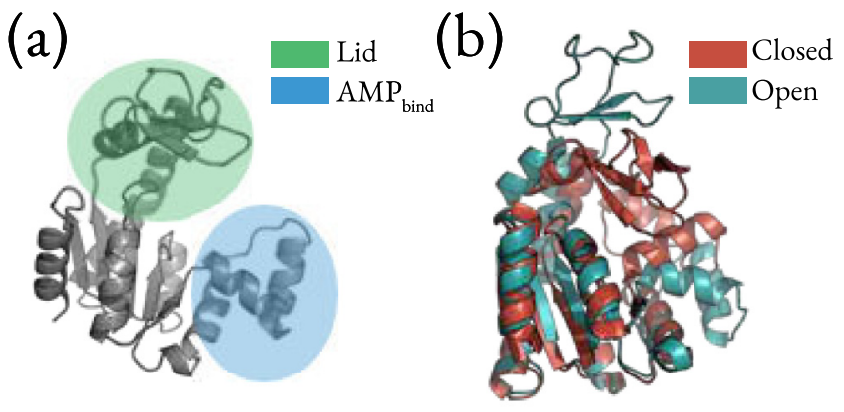

(c)

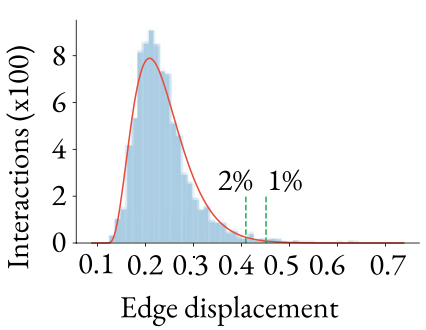

(d)

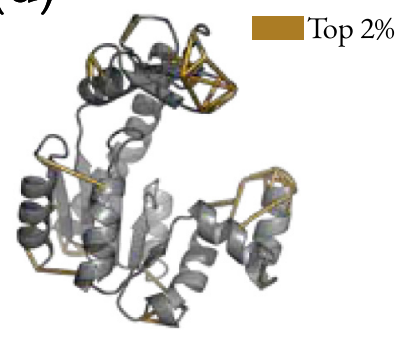

FIG. 4. (a) Structure of ADK from Escherichia coli (open conformation, PDB: $4 \mathrm{AKE}$ ), with the lid and $\mathrm{AMP}_{\text {bind }}$ domains highlighted. (b) Closed (1AKE) and open (4AKE) forms of ADK showing that the main differences are in the lid and $\mathrm{AMP}_{\text {bind }}$ domains. (c) The distribution of average edge displacements computed for open ADK (4AKE). (d) The top $2 \%$ residue-residue interactions with highest displacements are concentrated in the lid and $\mathrm{AMP}_{\text {bind }}$ domains. Note that the viewpoint of the structures in (b) has been changed slightly relative to (a) and (d), so as to facilitate the visualization of the differences between the open and closed conformations.

matrix) we can see that the interactions with the greatest average extension are also those with the lowest mechanical embeddedness, demonstrating a conceptual link to the network theory interpretation of protein structure.

\section{CONCLUSIONS}

In conclusion, we have presented here a framework for the study of geometric elastic network models in $d$-dimensional space through an alternative formulation in the edge space. The edge space is often more natural than the dual node space, as it allows the direct description of interactions and constraints, with their associated energies (or costs). By conveniently working with the internal coordinates of the network, there is no need to consider rigid motions or to arbitrarily "pin" nodes. In many systems, such as proteins, it is changes in the interactions that are of interest rather than the nodes themselves, and optimization problems involving edge variables are more naturally dealt with using an edge-based framework.

\section{ACKNOWLEDGMENTS}

This work was funded by an Engineering and Physical Sciences Research Council (EPSRC) Centre for Doctoral Training $\mathrm{PhD}$ Studentship from the Institute of Chemical Biology 
(Imperial College London) awarded to M.H. M.B. acknowledges funding from the EPSRC Project No. EP/N014529/1 supporting the EPSRC Centre for Mathematics of Precision Healthcare. We thank J. Costa for his help and insight with the infinitesimal rigidity algorithm.

\section{APPENDIX A: DERIVATION OF THE GEOMETRIC INCIDENCE MATRIX FOR THE TWO-CENTER INTERACTIONS}

For a spring connecting two nodes $i$ and $j$ with initial positions $\mathbf{r}_{i, 0}$ and $\mathbf{r}_{j, 0}$ and final positions $\mathbf{r}_{i}$ and $\mathbf{r}_{j}$, let $\mathbf{u}_{i}$ and $\mathbf{u}_{j}$ be the displacement of each node. We denote the final node positions by

$$
\begin{aligned}
\mathbf{r}_{i} & =\mathbf{r}_{i, 0}+\mathbf{u}_{i}, \\
\mathbf{r}_{j} & =\mathbf{r}_{j, 0}+\mathbf{u}_{j}
\end{aligned}
$$

with the vectors describing the springs before and after the extension (or compression) being

$$
\begin{aligned}
\mathbf{r}_{i j, 0} & =\mathbf{r}_{j, 0}-\mathbf{r}_{i, 0}, \\
\mathbf{r}_{i j} & =\mathbf{r}_{j}-\mathbf{r}_{i} .
\end{aligned}
$$

Consider the extension of an edge written in terms of the displacements of its associated nodes:

$$
e_{i j}=\left|\mathbf{r}_{i j}\right|-\left|\mathbf{r}_{i j, 0}\right| \text {. }
$$

We first expand $\left|\mathbf{r}_{i j, 0}\right|$,

$$
\left|\mathbf{r}_{i j, 0}\right|^{2}=\mathbf{r}_{i j, 0} \cdot \mathbf{r}_{i j, 0}=\left(\mathbf{r}_{j, 0}-\mathbf{r}_{j, 0}\right) \cdot\left(\mathbf{r}_{j, 0}-\mathbf{r}_{j, 0}\right),
$$

then expand $\left|\mathbf{r}_{i j}\right|$

$$
\begin{aligned}
\left|\mathbf{r}_{i j}\right|^{2} & =\mathbf{r}_{i j} \cdot \mathbf{r}_{i j}=\left|\mathbf{r}_{j, 0}+\mathbf{u}_{j}-\mathbf{r}_{i, 0}-\mathbf{u}_{i}\right|^{2} \\
& =\left|\left(\mathbf{r}_{j, 0}-\mathbf{r}_{i, 0}\right)+\left(\mathbf{u}_{j}-\mathbf{u}_{i}\right)\right|^{2} .
\end{aligned}
$$

Using Eq. (A4), we substitute terms

$$
\begin{aligned}
\left|\mathbf{r}_{i j}\right|^{2} & =\left|\mathbf{r}_{i j, 0}\right|^{2}+2 \mathbf{r}_{i j, 0}\left(\mathbf{u}_{j}-\mathbf{u}_{i}\right) \\
& =\left|\mathbf{r}_{i j, 0}\right|^{2}+2\left|\mathbf{r}_{i j, 0}\right| \frac{\mathbf{r}_{i j, 0}}{\left|\mathbf{r}_{i j, 0}\right|}\left(\mathbf{u}_{j}-\mathbf{u}_{i}\right) .
\end{aligned}
$$

We can complete the square

$$
\left|\mathbf{r}_{i j}\right|^{2}=\left(\left|\mathbf{r}_{i j, 0}\right|+\frac{\mathbf{r}_{i j, 0}}{\left|\mathbf{r}_{i j, 0}\right|}\left(\mathbf{u}_{j}-\mathbf{u}_{i}\right)\right)^{2}+\Theta\left(\mathbf{u}^{2}\right)
$$

and make a linear approximation by dropping nonlinear terms and square rooting both sides:

$$
\left|\mathbf{r}_{i j}\right|=\left|\mathbf{r}_{i j, 0}\right|+\frac{\mathbf{r}_{i j, 0}}{\left|\mathbf{r}_{i j, 0}\right|}\left(\mathbf{u}_{j}-\mathbf{u}_{i}\right) .
$$

By referring to Eq. (A3), we now have an expression for the change in spring length in terms of the node displacements

$$
e_{i j}=\frac{1}{\left|\mathbf{r}_{i j, 0}\right|} \mathbf{r}_{i j, 0} \cdot\left(\mathbf{u}_{i}-\mathbf{u}_{j}\right),
$$

which can be expressed in vector form as

$$
e_{i j}=\frac{1}{\left|\mathbf{r}_{i j, 0}\right|}\left[(1,-1) \otimes \mathbf{r}_{i j, 0}^{T}\right]\left(\begin{array}{c}
\mathbf{u}_{i} \\
\mathbf{u}_{j}
\end{array}\right) .
$$

Here, we have shown the expression for an isolated spring, For a spring in a network, the elements of the vectors relating

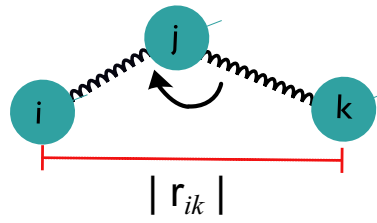

FIG. 5. Schematic for the derivation of the three-center (angle) interaction, where the two-center bond lengths $(i j),(j k)$ are kept constant and we compute the extension $i-k$ under those constraints.

to nodes not involved with the spring would be zero so that our geometric incidence matrix $\mathcal{B}$ has rows of the form (4).

\section{APPENDIX B: DERIVATION OF THE THREE-CENTER TERMS}

We wish to find an expression for the change in length of the distance $i-k$,

$$
e_{i k}=\left|\mathbf{r}_{i k}\right|-\left|\mathbf{r}_{i k, 0}\right|,
$$

in terms of the node displacements $\mathbf{u}_{i}, \mathbf{u}_{j}$, and $\mathbf{u}_{k}$ under the assumption that the edge distances are fixed (Fig. 5). The initial distance, using the cosine rule, is

$$
\left|\mathbf{r}_{i k, 0}\right|^{2}=\left|\mathbf{r}_{i j, 0}\right|^{2}+\left|\mathbf{r}_{j k, 0}\right|^{2}-2\left|\mathbf{r}_{i k, 0}\right|\left|\mathbf{r}_{j k, 0}\right| \cos \theta_{0}
$$

and likewise the distance after the perturbation of the three nodes is

$$
\left|\mathbf{r}_{i k}\right|^{2}=\left|\mathbf{r}_{i j}\right|^{2}+\left|\mathbf{r}_{j k}\right|^{2}-2\left|\mathbf{r}_{i k}\right|\left|\mathbf{r}_{j k}\right| \cos \theta .
$$

We apply the constraints $\left|\mathbf{r}_{i j}\right|=\left|\mathbf{r}_{i j, 0}\right|$ and $\left|\mathbf{r}_{j k, 0}\right|=\left|\mathbf{r}_{j k, 0}\right|$ as we are interested only in the change in angle, not in any two-center changes. We can then substitute terms from (B2) into (B3):

$$
\begin{aligned}
\left|\mathbf{r}_{i k}\right|^{2} & =\left|\mathbf{r}_{i k, 0}\right|^{2}+2\left|\mathbf{r}_{i k, 0}\right|\left|\mathbf{r}_{j k, 0}\right| \cos \theta_{0}-2\left|\mathbf{r}_{i k, 0}\right|\left|\mathbf{r}_{j k, 0}\right| \cos \theta \\
& =\left|\mathbf{r}_{i k, 0}\right|^{2}-2\left|\mathbf{r}_{i k, 0}\right|\left|\mathbf{r}_{j k, 0}\right|\left(\cos \theta-\cos \theta_{0}\right) .
\end{aligned}
$$

We can rewrite this expression as

$$
\left|\mathbf{r}_{i k}\right|^{2}=\left|\mathbf{r}_{i k, 0}\right|^{2}-2\left|\mathbf{r}_{i k, 0}\right|\left(\frac{\left|\mathbf{r}_{i j, 0}\right|\left|\mathbf{r}_{j k, 0}\right|}{\left|\mathbf{r}_{i k, 0}\right|}\left(\cos \theta-\cos \theta_{0}\right)\right)
$$

so that we can complete the square:

$$
\left|\mathbf{r}_{i k}\right|^{2}=\left(\left|\mathbf{r}_{i k}\right|-\frac{\left|\mathbf{r}_{i j, 0}\right|\left|\mathbf{r}_{j k, 0}\right|}{\left|\mathbf{r}_{i k, 0}\right|}\left(\cos \theta-\cos \theta_{0}\right)\right)^{2}+\Theta(|\mathbf{r}|)^{2} .
$$

By ignoring nonlinear terms and square rooting both sides can then write our extension from Eq. (B1) in terms of the initial and final angles:

$$
e_{i k}=\left(\frac{\left|\mathbf{r}_{i j, 0}\right|\left|\mathbf{r}_{j k, 0}\right|}{\left|\mathbf{r}_{i k, 0}\right|}\left(\cos \theta-\cos \theta_{0}\right)\right) .
$$

However, we wish to derive the extension in terms of node displacements (in Cartesian coordinates) and so substitute using the definition of the dot product:

$$
e_{i k}=\frac{1}{\left|\mathbf{r}_{i k}\right|}\left[\left(\mathbf{r}_{i j} \cdot \mathbf{r}_{j k}\right)-\left(\mathbf{r}_{i j, 0} \cdot \mathbf{r}_{j k, 0}\right)\right],
$$

where we have again used the fact that the two bonds have not changed length. We now define the displacements of the nodes 
in terms of the bond vectors before and after perturbation:

$$
\begin{aligned}
\mathbf{u}_{i} & =\mathbf{r}_{i}-\mathbf{r}_{i, 0}, \\
\mathbf{u}_{j} & =\mathbf{r}_{j}-\mathbf{r}_{j, 0}, \\
\mathbf{u}_{k} & =\mathbf{r}_{k}-\mathbf{r}_{k, 0} .
\end{aligned}
$$

We now expand out Eq. (B5):

$$
\begin{aligned}
e_{i k}= & \frac{1}{\left|\mathbf{r}_{i k}\right|}\left(\mathbf{r}_{i} \cdot \mathbf{r}_{j}-\mathbf{r}_{i} \cdot \mathbf{r}_{k}-\mathbf{r}_{j} \cdot \mathbf{r}_{j}+\mathbf{r}_{j} \cdot \mathbf{r}_{k}\right. \\
& \left.-\mathbf{r}_{i, 0} \cdot \mathbf{r}_{j, 0}+\mathbf{r}_{i, 0} \cdot \mathbf{r}_{k, 0}+\mathbf{r}_{j, 0} \cdot \mathbf{r}_{j, 0}-\mathbf{r}_{j, 0} \cdot \mathbf{r}_{k, 0}\right) .
\end{aligned}
$$

Substituting terms from (B6), we drop the nonlinear terms in the second line of (B7) to get

$$
\begin{aligned}
e_{i k}= & \left(\mathbf{r}_{i, 0}+\mathbf{u}_{i}\right) \cdot\left(\mathbf{r}_{j, 0}+\mathbf{u}_{j}\right)-\left(\mathbf{r}_{i, 0}+\mathbf{u}_{i}\right) \cdot\left(\mathbf{r}_{k, 0}+\mathbf{u}_{k}\right) \\
& -\left(\mathbf{r}_{j, 0}+\mathbf{u}_{j}\right) \cdot\left(\mathbf{r}_{j, 0}+\mathbf{u}_{j}\right)+\left(\mathbf{r}_{j, 0}+\mathbf{u}_{j}\right) \cdot\left(\mathbf{r}_{k, 0}+\mathbf{u}_{k}\right) .
\end{aligned}
$$

Now, we again drop nonlinear terms that result from the expansion of each of the dot products to give

$$
\begin{aligned}
e_{i k}= & \frac{1}{\left|\mathbf{r}_{i k}\right|}\left(\mathbf{r}_{i, 0} \cdot \mathbf{u}_{j}+\mathbf{r}_{j, 0} \cdot \mathbf{u}_{i}-\mathbf{r}_{i, 0} \cdot \mathbf{u}_{k}-\mathbf{r}_{k, 0} \cdot \mathbf{u}_{i}\right. \\
& \left.-\mathbf{r}_{j, 0} \cdot \mathbf{u}_{j}-\mathbf{r}_{j, 0} \cdot \mathbf{u}_{j}+\mathbf{r}_{j, 0} \cdot \mathbf{u}_{k}+\mathbf{r}_{k, 0} \cdot \mathbf{u}_{j}\right)
\end{aligned}
$$

which can be written more compactly as

$$
\begin{aligned}
e_{i k}= & \left(\mathbf{r}_{j, 0}-\mathbf{r}_{k, 0}\right) \mathbf{u}_{i}-\left(\left(\mathbf{r}_{j, 0}-\mathbf{r}_{i, 0}\right)+\left(\mathbf{r}_{j, 0}-\mathbf{r}_{k, 0}\right)\right) \mathbf{u}_{j} \\
& +\left(\mathbf{r}_{j, 0}-\mathbf{r}_{i, 0}\right) \mathbf{u}_{k}
\end{aligned}
$$

or, in vector form, as

$$
e_{i k}=\frac{1}{\left|\mathbf{r}_{i k, 0}\right|}\left(\mathbf{r}_{j k, 0}^{T}, \quad\left(\mathbf{r}_{i j, 0}^{T}-\mathbf{r}_{j k, 0}^{T}\right), \quad-\mathbf{r}_{i j, 0}^{T}\right)\left(\begin{array}{c}
\mathbf{u}_{i} \\
\mathbf{u}_{j} \\
\mathbf{u}_{k}
\end{array}\right) .
$$

As for the two-center case, each row of $\mathcal{B}_{\text {angle }}$, the geometric incidence matrix for the three-center interactions, has the form (B9) but with zeros in the entries relating to nodes not involved in the corresponding interaction.

The stiffness matrix for the three-center interactions can then be constructed similarly:

$$
\mathbf{K}_{\text {angle }}=\mathcal{B}_{\text {angle }} \mathbf{G}_{\text {angle }} \mathcal{B}_{\text {angle }}^{T},
$$

with $\mathbf{G}_{\text {angle }}$ the diagonal matrix containing the spring constants for the three-center ("angle") interactions.

\section{APPENDIX C: EXPRESSION FOR THE FOUR-CENTER TERMS}

The derivation for the four-center (or dihedral) terms is similar to the three-center case, where we compute the extension in the length between $i-l$ while keeping constant the two angular terms $(i j k)$ and $(j k l)$, as well as each of the three bond lengths $(i j),(j k),(k l)$ (Fig. 6). This leads to the expression

$$
\begin{aligned}
e_{i l}= & \frac{1}{\left|\mathbf{r}_{i l, 0}\right|}\left[\left(\mathbf{r}_{j l, 0}+\mathbf{r}_{i k, 0}\right) \cdot \mathbf{u}_{i}+\left(\mathbf{r}_{i j, 0}+\mathbf{r}_{j k, 0}+\mathbf{r}_{j l, 0}\right) \cdot \mathbf{u}_{j}\right. \\
& \left.-\left(\mathbf{r}_{j k, 0}+\mathbf{r}_{k l, 0}+\mathbf{r}_{i k, 0}\right) \cdot \mathbf{u}_{k}-\left(\mathbf{r}_{j l, 0}+\mathbf{r}_{i k, 0}\right) \cdot \mathbf{u}_{l}\right],(\mathrm{C} 1)
\end{aligned}
$$

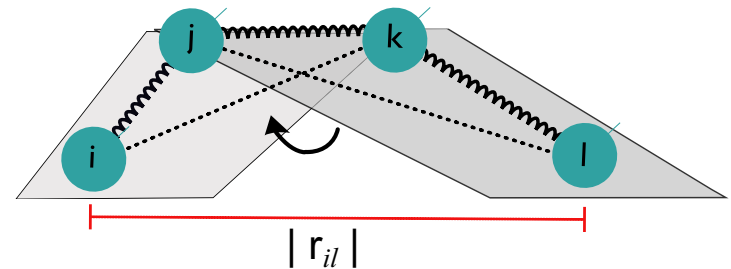

FIG. 6. Schematic for the derivation of the dihedral interaction: the three two-center bond lengths $(i j),(j k),(k l)$ remain constant, as well as the two three-center angle interactions $(i j k)$ and $(j k l)$ marked with the dashed lines. We compute the extension $i-l$ under those constraints.

which, by using $\mathbf{r}_{j l, 0}=\mathbf{r}_{j k, 0}+\mathbf{r}_{k l, 0}$ and $\mathbf{r}_{i k, 0}=\mathbf{r}_{i j, 0}+\mathbf{r}_{j k, 0}$, is transformed into

$$
\begin{aligned}
e_{i l}= & \frac{1}{\left|\mathbf{r}_{i l, 0}\right|}\left[\left(\begin{array}{lll}
1, & 1,-1, & -1
\end{array}\right)\right. \\
& \left.\otimes\left(\mathbf{r}_{i j, 0}^{T}+2 \mathbf{r}_{j k, 0}^{T}+\mathbf{r}_{k l, 0}^{T}\right)\right]\left(\begin{array}{l}
\mathbf{u}_{i} \\
\mathbf{u}_{j} \\
\mathbf{u}_{k} \\
\mathbf{u}_{l}
\end{array}\right) .
\end{aligned}
$$

\section{APPENDIX D: DEFINITION OF THE RIGIDITY MATRIX}

Here, we summarize standard calculations covered in Refs. [26,27,40]. In this Appendix and in the subsequent Appendix E, we adopt the usual notation in rigidity theory, where the positions of the points are represented as $\mathbf{p}_{i}$, and the system is defined by a set $\mathcal{M}$ of $M$ distance constraints. Note that in the rest of the paper, $\mathbf{p}_{i}$ are denoted as $\mathbf{r}_{i}$, and the $M$ constraints correspond to the $E$ edges of the graph encapsulating the interactions.

The rigidity problem for $N$ points $\mathbf{p}_{i} \in \mathbb{R}^{d}, i=1, \ldots, N$, with a set $\mathcal{M}$ of distance constraints $c_{\alpha}, \alpha=1, \ldots, M$, is given explicitly by the following set of $M$ equations:

$$
\left|\mathbf{p}_{i}-\mathbf{p}_{j}\right|^{2}=c_{i j}=: c_{\alpha}, \quad \alpha=(i j) \in \mathcal{M},
$$

where $\mathbf{p}_{i}$ is the $3 \times 1$ position vector of node $i$. Solving this set of $M$ nonlinear equations directly is usually infeasible for anything but very small systems. An alternative approach is infinitesimal rigidity, which considers infinitesimal violations of the equilibrium conditions of (D1).

Taking the derivative of both sides of (D1) with respect to time $t$ for all constrained pairs, we get

$$
\left(\mathbf{p}_{i}-\mathbf{p}_{j}\right) \cdot\left(\mathbf{u}_{i}-\mathbf{u}_{j}\right)=0, \quad(i j) \in \mathcal{M}
$$

with $\mathbf{u}_{i}=d \mathbf{p}_{i} / d t$. We then expand out the brackets:

$$
\left(\mathbf{p}_{i}-\mathbf{p}_{j}\right) \mathbf{u}_{i}-\left(\mathbf{p}_{i}-\mathbf{p}_{j}\right) \mathbf{u}_{j}=0,
$$

and rewrite in vector form

$$
\mathbf{R u}=\mathbf{0} .
$$

The $M \times N d$ matrix $\mathbf{R}$ is called the rigidity matrix and each row represents a single constraint. For example, a three-node system with each pair of nodes joined by an edge would have the rigidity matrix [27]

$$
\mathbf{R}=\left(\begin{array}{ccc}
\mathbf{p}_{1}-\mathbf{p}_{2}, & \mathbf{p}_{2}-\mathbf{p}_{1}, & \mathbf{0} \\
\mathbf{0}, & \mathbf{p}_{2}-\mathbf{p}_{3}, & \mathbf{p}_{3}-\mathbf{p}_{2} \\
\mathbf{p}_{1}-\mathbf{p}_{3}, & \mathbf{0}, & \mathbf{p}_{3}-\mathbf{p}_{1}
\end{array}\right) .
$$



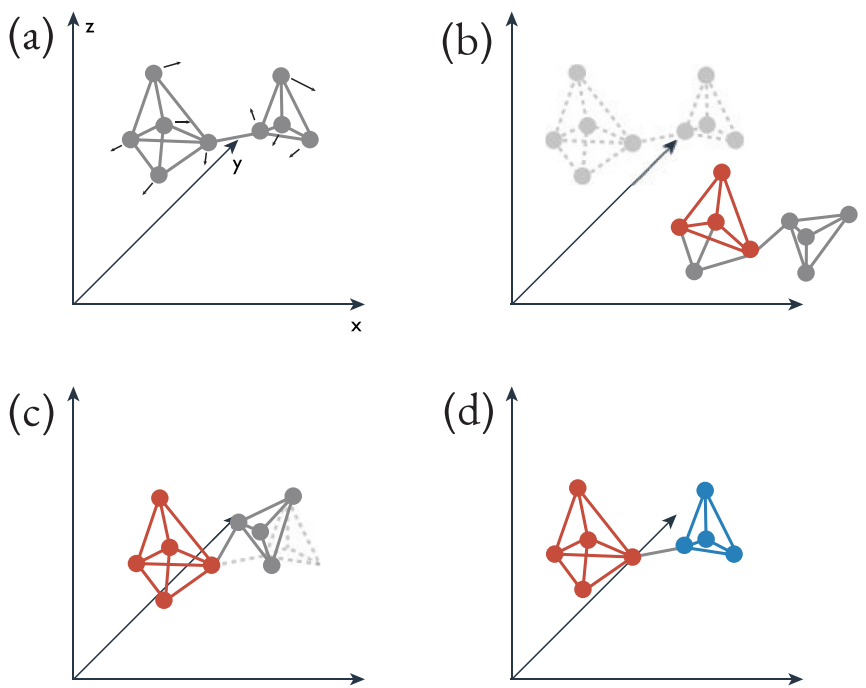

FIG. 7. Rigid cluster decomposition algorithm using infinitesimal rigidity [27]. For each trivial infinitesimal motion, such as the one in (a), the atoms are moved by a small distance along each $3 N \times 1$ vector to a new position (b). A rigid tetrahedron of atoms is selected in the new position then in (c) this is moved back to its original position. Any atoms that also return to their original position at the same time (for all infinitesimal motions) are part of the same cluster. (d) The process is repeated until all atoms are clustered into rigid regions or are assigned as floppy.

The infinitesimal rigidity properties follow from examining the null space of $\mathbf{R}$. Hence, these properties are an intrinsic property of the structure, and are independent of the environment or the friction terms. From (D5), it follows immediately that the geometric incidence matrix is a scaled version of $\mathbf{R}$.

\section{APPENDIX E: ALGORITHM FOR RIGID CLUSTER DECOMPOSITION USING INFINITESIMAL RIGIDITY}

The following algorithm was introduced in Ref. [27] and is summarized here for completeness. We use it to obtain the rigid clusters shown in Fig. 3.

The steps of the algorithm are as follows (see Fig. 7):

(1) Identify a set of four atoms $\mathcal{T}$ that form a fully connected tetrahedron.

(2) Translate the coordinate frame to the center of the set $\mathcal{T}:$

$$
\mathbf{p}_{k}:=\mathbf{p}_{k}-\frac{1}{4} \sum_{k \in \mathcal{T}} \mathbf{p}_{k}
$$

(3) Transform the three coordinate axes so that they correspond to the principal axes of the set $\mathcal{T}$ :

$$
\mathbf{p}_{k}:=\mathbf{S} \mathbf{p}_{k} .
$$

$\mathbf{S}$ is the rotation matrix whose rows are the eigenvectors of the matrix I:

$$
\mathbf{I}_{\alpha \beta}=\sum_{k \in T}\left(\left|\mathbf{p}_{k}\right| \delta_{\alpha \beta}-\mathbf{p}_{k \alpha} \mathbf{p}_{k \beta}\right), \quad \text { where }(\alpha, \beta)=(x, y, z) .
$$

(4) Generate the trivial motions in this new coordinate frame: three rotations $\left(\mathbf{r}_{k}^{x}, \mathbf{r}_{k}^{y}, \mathbf{r}_{k}^{z}\right)$ and three translations

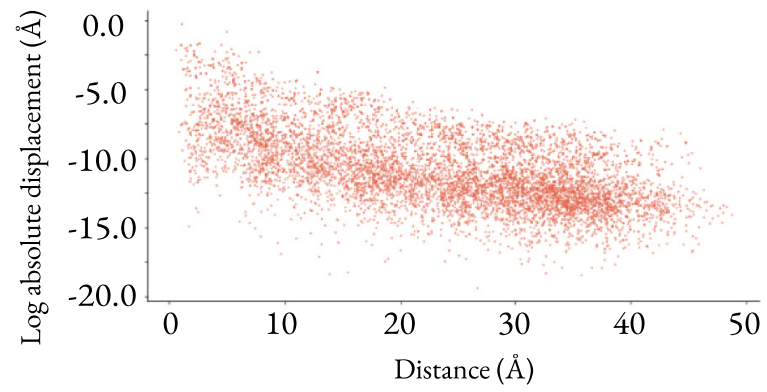

FIG. 8. The log absolute extension of interactions decreases linearly as a function of distance from the allosteric source site with slope -0.142 (correlation coefficient $=-0.603$, standard error $=0.0022$ ), i.e., the effect of the perturbation decays exponentially away from the allosteric site.

$\left(\mathbf{t}_{k}^{x}, \mathbf{t}_{k}^{y}, \mathbf{t}_{k}^{z}\right)$ for all of the atoms of the structure:

$$
\mathbf{r}_{k}^{\alpha}=\mathbf{p}_{k} \times \widehat{\mathbf{e}^{\alpha}} ; \quad \mathbf{t}_{k}^{\alpha}=\widehat{\mathbf{e}^{\alpha}} .
$$

(5) Transform the trivial motions back into the starting coordinate frame

$$
\mathbf{r}_{k}^{\alpha}:=\mathbf{S}^{T} \mathbf{r}_{k}^{\alpha} ; \quad \mathbf{t}_{k}^{\alpha}:=\mathbf{S}^{T} \mathbf{t}_{k}^{\alpha} .
$$

(6) Compile the trivial motions for each atom into column vectors so we have three $3 N$-dimensional translations $\mathbf{t}^{\alpha}$ and three rotations $\mathbf{r}^{\alpha}$. Normalize each of these trivial motions:

$$
\mathbf{r}^{\alpha}:=\frac{\mathbf{r}^{\alpha}}{\left|\mathbf{r}_{\mathbf{T}}^{\alpha}\right|} ; \quad \mathbf{t}^{\alpha}:=\frac{\mathbf{t}^{\alpha}}{\left|\mathbf{t}_{\mathbf{T}}^{\alpha}\right|}
$$

using the magnitude of the 12-dimensional vectors associated with the set $\mathcal{T}$. Now, the set of six 12-dimensional trivial motions of the set $\mathcal{T}$ are orthonormal.

(7) The set of displacements of each of the atoms relative to the set $\mathcal{T}$ can then be calculated by returning the set $\mathcal{T}$ to its initial position

$$
\Delta \mathbf{p}^{\gamma}=\mathbf{q}^{\gamma}-\sum_{\alpha}\left(\mathbf{q}_{T}^{\gamma} \cdot \mathbf{r}(t)^{\alpha}\right) r^{\alpha}-\sum_{\alpha}\left(\mathbf{q}_{T}^{\gamma} \cdot \mathbf{t}_{T}^{\alpha}\right) \mathbf{t}^{\alpha},
$$

where we now use $\gamma$ additionally index over the set of trivial motions.

(8) For each atom, calculate its absolute displacement in space away from its initial position due to the infinitesimal motions. If the maximum displacement of the atom over the entire set of infinitesimal motions is below a chosen small threshold value, then we say that atom is part of the same rigid cluster as the set $\mathcal{T}$ :

$$
\max _{\gamma}\left|\Delta \mathbf{p}_{k}^{\gamma}\right|<\delta
$$

where $\delta$ is a cutoff to account for floating point rounding error. Here, we use $\delta=10^{-4}$.

\section{APPENDIX F: EDGE DISPLACEMENT DECAYS EXPONENTIALLY WITH DISTANCE}

In all of the protein structures studied, it was found that the absolute extension of the springs decreased exponentially with increasing distance of the spring from the perturbation site, as shown in Fig. 8. 
[1] H. He and M. F. Thorpe, Phys. Rev. Lett. 54, 2107 (1985).

[2] V. V. Palyulin, C. Ness, R. Milkus, R. M. Elder, T. W. Sirk, and A. Zaccone, Soft Matter 14, 8475 (2018).

[3] I. B. Bischofs, F. Klein, D. Lehnert, M. Bastmeyer, and U. S. Schwarz, Biophys. J. 95, 3488 (2008).

[4] L. Yan, G. Düring, and M. Wyart, Proc. Natl. Acad. Sci. USA 110, 6307 (2013).

[5] L. Yan, R. Ravasio, C. Brito, and M. Wyart, Proc. Natl. Acad. Sci. USA 114, 2526 (2016).

[6] M. M. Tirion, Phys. Rev. Lett. 77, 1905 (1996).

[7] K. Henzler-Wildman and D. Kern, Nature (London) 450, 964 (2007).

[8] U. Bastolla, Wiley Interdiscip. Rev.: Comput. Mol. Sci. 4, 488 (2014).

[9] J. R. López-Blanco, O. Miyashita, F. Tama, and P. Chacón, in eLS (Wiley, Hoboken, NJ, 2014).

[10] I. Bahar, A. R. Atilgan, and B. Erman, Folding Des. 2, 173 (1997).

[11] L. Yang, G. Song, and R. L. Jernigan, Proc. Natl. Acad. Sci. USA 106, 12347 (2009).

[12] G. Strang, Introduction to Applied Mathematics (WellesleyCambridge Press, Wellesley, MA, 1986).

[13] B. R. Amor, M. T. Schaub, S. N. Yaliraki, and M. Barahona, Nat. Commun. 7, 12477 (2016).

[14] M. T. Schaub, J. Lehmann, S. N. Yaliraki, and M. Barahona, Network Sci. 2, 66 (2014).

[15] M. Hodges, M. Barahona, and S. N. Yaliraki, Sci. Rep. 8, 11079 (2018).

[16] M. F. Thorpe, Phys. Biol. 4, 60 (2007).

[17] M. Born and K. Huang, Dynamical Theory of Crystal Lattices (Clarendon, Oxford, 1954).

[18] S. Feng, P. N. Sen, B. I. Halperin, and C. J. Lobb, Phys. Rev. B 30, 5386 (1984).

[19] A. Zaccone and E. Scossa-Romano, Phys. Rev. B 83, 184205 (2011).

[20] A. Ghosh, S. Boyd, and A. Saberi, SIAM Rev. 50, 37 (2008).

[21] D. J. Klein and M. Randić, J. Math. Chem. 12, 81 (1993).
[22] K. Hinsen, A. J. Petrescu, S. Dellerue, M. C. Bellissent-Funel, and G. R. Kneller, Chem. Phys. 261, 25 (2000).

[23] C. L. McClendon, A. P. Kornev, M. K. Gilson, and S. S. Taylor, Proc. Natl. Acad. Sci. USA 111, E4623 (2014).

[24] N. T. Doncheva, Y. Assenov, F. S. Domingues, and M. Albrecht, Nat. Protoc. 7, 670 (2012).

[25] A. A. S. T. Ribeiro and V. Ortiz, J. Chem. Theory Comput. 10, 1762 (2014).

[26] W. Whiteley, Phys. Biol. 2, S116 (2005).

[27] J. R. Costa, Ph.D. thesis, Imperial College, London, 2008.

[28] D. J. Jacobs, A. J. Rader, L. A. Kuhn, and M. F. Thorpe, Proteins 44, 150 (2001).

[29] D. J. Jacobs and M. F. Thorpe, Computer-implemented system for analyzing rigidity of substructures within a macromolecule, US Patent No. 6014449 (1998).

[30] R. Milkus, C. Ness, V. V. Palyulin, J. Weber, A. Lapkin, and A. Zaccone, Macromolecules 51, 1559 (2018).

[31] C. J. Tsai and R. Nussinov, PLoS Comput. Biol. 10, e1003394 (2014).

[32] A. A. S. T. Ribeiro and V. Ortiz, Chem. Rev. 116, 6488 (2016).

[33] L.-P. Wang, K. A. McKiernan, J. Gomes, K. A. Beauchamp, T. Head-Gordon, J. E. Rice, W. C. Swope, T. J. Martínez, and V. S. Pande, J. Phys. Chem. B 121, 4023 (2017).

[34] J. D. Sadowsky, M. A. Burlingame, D. W. Wolan, C. L. McClendon, M. P. Jacobson, and J. A. Wells, Proc. Natl. Acad. Sci. USA 108, 6056 (2011).

[35] C. W. Müller, G. J. Schlauderer, J. Reinstein, and G. E. Schulz, Structure 4, 147 (1996).

[36] A. R. Atilgan, S. R. Durell, R. L. Jernigan, M. C. Demirel, O. Keskin, and I. Bahar, Biophys. J. 80, 505 (2001).

[37] M. R. Mitchell, T. Tlusty, and S. Leibler, Proc. Natl. Acad. Sci. USA 113, E5847 (2016).

[38] J. Günther, A. Bergner, M. Hendlich, and G. Klebe, J. Mol. Biol. 326, 621 (2003).

[39] Y. Wang and L. Makowski, Proteins 86, 332 (2018).

[40] J. R. Costa and S. N. Yaliraki, J. Phys. Chem. B 110, 18981 (2006). 